\title{
Applying the Virtual Input-Output Method to the Identification of Key Nodes in Busy Traffic Network
}

\author{
Fan Yang $\left(\mathbb{D},{ }^{1}\right.$ Fei Yan, ${ }^{1}$ Chikun Zhang, ${ }^{2}$ Xiaoying Tang $\left(\mathbb{D},{ }^{3}\right.$ Jianchang Li $\left(\mathbb{D},{ }^{4}\right.$ Xindan Zhang, ${ }^{5}$ \\ and Yingxin Gan' \\ ${ }^{1}$ School of Management, Xi'an Polytechnic University, Xi'an, Shaanxi 710048, China \\ ${ }^{2}$ Department of Architecture and Civil Engineering, City University of Hong Kong, Kowloon 999077, Hong Kong \\ ${ }^{3}$ Department of Engineering Management, School of Civil Engineering, Central South University, Changsha, \\ Hunan 410075, China \\ ${ }^{4}$ Department of Financial Mathematics, School of Science, Xi'an Jiaotong-Liverpool University, Suzhou, Jiangsu 215123, China \\ ${ }^{5}$ Chang'an Dublin International College of Transportation at Chang'an University, Chang'an University, Xi'an, \\ Shaanxi 710021, China \\ ${ }^{6}$ Zhengping Road \& Bridge Construction Co.Ltd.,, Xining, Qinghai 810008, China
}

Correspondence should be addressed to Xiaoying Tang; xiaoyingtang311@csu.edu.cn

Received 7 February 2021; Revised 10 March 2021; Accepted 2 April 2021; Published 13 April 2021

Academic Editor: Muhammad Javaid

Copyright (c) 2021 Fan Yang et al. This is an open access article distributed under the Creative Commons Attribution License, which permits unrestricted use, distribution, and reproduction in any medium, provided the original work is properly cited.

\begin{abstract}
How to identify the key nodes effectively in urban traffic networks to achieve the equitable resource allocation face to the complex traffic network? This issue needs to be solved in current traffic management. This study considered the urban traffic network topology and network traffic status, put forward an improved model based on the economics of the input-output method by introducing a virtual node to the selected network set up with the flow of urban traffic network, sensor nodes by Leontief inverse matrix calculation coefficient to determine node importance, according to the node importance to deliberate attack traffic network to analyze its robustness, to test the accuracy and practicability of the method. The results show that this improved method adopted to measure the importance of traffic nodes from the global scope has the advantages of fast calculation and simple process and provides a more reliable basis for rational allocation of transport resources.
\end{abstract}

\section{Introduction}

The topological characteristics of urban integrated traffic networks could determine the importance of nodes in the network, and the identification of the significance of different nodes in urban traffic networks is crucial to the study of urban traffic resilience and the reduction of the operational efficiency of urban traffic networks under unexpected conditions. With the accelerating urbanization process, the traffic volume of the urban road network has increased dramatically. Therefore, it is of great significance to assess the importance of nodes in the integrated urban traffic network for urban transportation management.

There have been many research results in the study of the importance of nodes in complex networks and transportation networks. White \& Smyth defined and studied the relative importance of nodes in the network, and constructed a basic theoretical framework about the importance of nodes in complex networks [1]. Chen et al. considered the information of nodes, the nearest neighbor nodes, and the next neighbor nodes in the network, and measured the importance of nodes in complex networks by defining multilevel neighbor information indicators [2]. Kitsak et al. conducted a study on the ranking index of node importance by considering the position of nodes in the whole network [3]. Chen \& Sun constructed an evaluation model combining node proximity and neighborhood criticality to examine the importance of complex network nodes [4]. Yu et al. built an attribute decision-based node importance evaluation model for complex networks, which can 
be adopted to identify the important nodes in complex networks [5]. Coscia et al. showed Node Vector Distance (NVD) problem has many significant applications and established a roadmap that can provide useful and computationally tractable solutions [6]. For the effective identification of key nodes in the transportation network, most of the existing research results are aimed at the complex and functional properties of traffic networks. For instance, Song et al. selected three evaluation indicators of connectivity, intermediate center, and proximity measuring the significance of urban roads to construct the urban road importance evaluation model [7]. Zhang \& Yao introduced the evaluation indexes to define the running state fragility and structural fragility of the urban road network [8]. Li et al. chose the global effectiveness as the index to recognize the key nodes and key sections of road networks based on the characteristics of multimodal composite traffic networks in urban clusters [9].

In the existing studies, the classical identification indicators of key nodes are more concerned with the topological characteristics of the traffic network, but the dynamics and overall aspects of urban traffic networks are less considered. This paper proposes an improved method to identify key nodes of urban traffic networks based on the theory of virtual input-output method combined with static and dynamic characteristics of urban traffic networks. This method is applied to actual cases for key node identification. In order to test the effectiveness of the improved virtual input-output method in identifying key nodes of urban traffic networks, the traffic network robustness is analyzed after the nodes are deliberately attacked according to the importance of the nodes. After testing, it can be concluded that the identification method of key nodes proposed in this study is simpler, more practical, and has higher accuracy.

\section{Evaluation Model of Importance of Urban Traffic Nodes Based on Input-Output Method}

2.1. Method for Identifying Importance of Urban Traffic Network Nodes. The urban transportation network is essentially a complex network with topological characteristics. Considering the node importance in urban transportation networks, the research focus should be on how to identify the importance of nodes. The classic identification methods of key nodes in the network are as follows: the degree centrality of the degree value of the network node, the betweenness centrality of the network node, the closeness centrality of the node in the network, and the node in the network compactness. These methods focus more on the topological characteristics of the network, which are used for identifying key nodes in the transportation network. When determining critical nodes, it is common to use parameters (e.g., saturation, capacity, and average latency time) for measurement. These parameters reflect the dynamic characteristics of distinct nodes in the urban transportation network. However, it is difficult to reflect the overall network structure and the interaction with nodes in the urban transportation network. These interactions are the key basis for determining the importance of urban transportation network nodes.

Based on the virtual input-output method, this research builds a new method of identifying key nodes of the urban transportation network, combined with the topological characteristics of the urban transportation network and realtime traffic volume. It can introduce a virtual node into the network created by urban traffic networks and sensor nodes. This method can effectively determine the importance of traffic nodes from the global scope and thus better allocate resources in the case of complex traffic networks. This is conducive to the government to propose dynamic policies which can better improve the stability of traffic [10]. The basis of this method is the economic input-output model, which applies the key industry identification method in the input-output model to the evaluation of the importance of urban transportation network nodes.

2.2. Economic Input-Output Model. The input-output model was first proposed by American economist Wassily Leontief, which reflects the flow and exchange of products and services between various industrial sectors in an economic system in a certain period [11]. According to the input and output of the industry, an input-output matrix can be obtained. The rows of the matrix represent the consumption or input of products produced by various sectors of the national economy, and the columns represent the output or value formation of products of numerous sectors. After calculation, it can intuitively reveal the relevance of diverse industries in economic activities [12].

Assume that there are $N$ departments in a certain economic field and $x_{i j}$ represents the value of intermediate products provided by the $i$ department to the $j$ department, according to the balanced relationship of the input-output model:

$$
\sum_{j=1}^{n} x_{i j}+Y_{i}=X_{i}, \quad i=1,2, \ldots, n
$$

The direct consumption coefficient can be obtained:

$$
a_{i j}=\frac{x_{i j}}{X_{j}}, \quad i, j=1,2, \ldots, n .
$$

It can be xpressed as a matrix:

$$
A=Q \times \widehat{X}^{-1},
$$

where 


$$
\begin{aligned}
A & =\left[\begin{array}{cccc}
a_{11} & a_{12} & \cdots & a_{1 n} \\
a_{21} & a_{22} & \cdots & a_{2 n} \\
\vdots & \vdots & & \vdots \\
a_{n 1} & a_{n 2} & \cdots & a_{n n}
\end{array}\right], \\
Q & =\left[\begin{array}{cccc}
x_{11} & x_{12} & \cdots & x_{1 n} \\
x_{21} & x_{22} & \cdots & x_{2 n} \\
\vdots & \vdots & & \vdots \\
x_{n 1} & x_{n 2} & \cdots & x_{n n}
\end{array}\right], \\
\widehat{X}^{-1} & =\left[\begin{array}{cccc}
\frac{1}{X_{1}} & 0 & \cdots & 0 \\
& & & \\
0 & \frac{1}{X_{2}} & \cdots & 0 \\
\vdots & \vdots & & \vdots \\
0 & 0 & \cdots & \frac{1}{X_{n}}
\end{array}\right] .
\end{aligned}
$$

According to formulas (1) and (2), the complete consumption coefficient can be obtained:

$$
b_{i j}=a_{i j}+\sum_{k=1}^{n} b_{i k} a_{k j}, \quad i, j=1,2, \ldots, n .
$$

It can be expressed in matrix form as shown below:

$$
B=(I-A)^{-1}-I \text {, }
$$

where

$$
B=\left[\begin{array}{cccc}
b_{11} & b_{12} & \cdots & b_{1 n} \\
b_{21} & b_{22} & \cdots & b_{2 n} \\
\vdots & \vdots & & \vdots \\
b_{n 1} & b_{n 2} & \cdots & b_{n n}
\end{array}\right] .
$$

Then $(I-A)^{-1}$ is called the Leontief inverse matrix.

Let $A_{i j}$ denote the coefficient of row $i$ and column $j$ in matrix $(I-A)^{-1}$; then the sensitivity coefficient $S_{i}$ can be obtained:

$$
S_{i}=\frac{(1 / n) \sum_{j=1}^{n} A_{i j}}{\left(1 / n^{2}\right) \sum_{i=1}^{n} \sum_{j=1}^{n} A_{i j}}, \quad(i, j=1,2, \ldots, n) .
$$

The sensitivity coefficient is an important concept in the input-output method. It can reflect the economic dependence and mutual influence between industries. The meaning of the inductance coefficient is the degree of demand induction that a certain department receives when each department produces a unit of the final product, that is, the output value provided by the department for other departments to produce the unit's ultimate product. The sensitivity coefficient reflects the degree of influence of a certain industry on the other industries in the economic system. The coefficient being greater than 1 can indicate that the demand sensitivity of the industry is higher than the industry average. On the contrary, when the inductance coefficient is less than 1 , it is lower than the industry average.

2.3. Improved Virtual Input-Output Method. In addition to the application in the economic field, the input-output model has also been extended to other noneconomic fields. Zhu et al. adopted the virtual input-output method to rank the importance of Chinese railway network nodes. Compared with other algorithms, the results are more in line with people's intuitive understanding of the importance of stations [13]. Shen et al. used the method of obtaining the inverse matrix of the input-output matrix of the closed flow network to calculate the influence of the nodes in the network and applied it to the research of evaluating the influence of the subject field [14].

In the traditional input-output model, when each industry produces one unit of the final product, an industry with a larger inductance coefficient needs to increase production more, and the more it reflects the status of the basic industry. When the economy grows rapidly, the industry would have the greatest demand pressure. However, the assessment standards which do not focus on the operation stage may cause inaccurate results [15]. In the calculation example of the virtual input-output method in this article, it can be understood that when each node in the road network increases the traffic volume by one unit, the large nodes need to increase the greater traffic volume to match it, and they receive influences from other sources. The pressure on the nodes will also increase.

This paper studies the urban transportation network with the virtual input-output method, which can be regarded as a flow network. A flow network is a directional weighted network. The connected edges between nodes represent the direction of network media flow, and the weight of the edges in the network represents the amount of traffic. The nodes of the flow network can be source, sink, or general path nodes [16]. The source is the node where the flow is generated, the sink is the node where the flow disappears, and the general road node plays the role of the passage. Streaming networks are similar to ordinary complex networks, and both can be expressed as matrices.

In a complex network, the elements of the matrix represent the connection between nodes $i$ and $j$ in the network. If the value is equal to 1 , it means that the two nodes are connected. Otherwise, they are not connected. In the streaming network, the expression form is the same, except $f_{i j}$ represents the size of the traffic from $i$ to $j$, and $f_{i j}$ may not be equal to $f_{j i}$. By using the virtual input-output method, the edges in the urban transportation network can be regarded as industrial connections in the input-output system, and the flow from one node to another node in the urban transportation network can be regarded as the inputoutput system provided by an industry an intermediate product in another industry. 
Due to the difference between urban transportation networks and industrial economics, when we apply the economics input-output method to the identification of key nodes in the transportation network, it is necessary to consider the urban transportation network as a nonclosed flow network, which has the problem of flow at the network boundary. Some activities in the close flow network might affect the basis of the local ecological system negatively [17]. In order to solve this problem, the author applied the method used in social network node influence ranking, adding a virtual node $n$ in the research object, allowing this node to be analyzed as a source and a sink of the research network simultaneously. The boundary nodes of the experimental network are bidirectionally connected [18]. The urban transportation network, after adding sources and sinks, provides the feasibility for the subsequent application of the input-output method to identify key nodes of the urban transportation network. Compared with the traditional method, the improved virtual input-output treats each node in the transportation network as each sector in this model and introduces virtual nodes to build a new urban transportation network structure. After calculating the inductance coefficient of a traffic network node, the importance of the node can be evaluated. Therefore, the robustness of the traffic network after failure can be analyzed and judged. The model is conducive to the rational allocation of traffic resources by the relevant departments.

\section{Case Study}

This paper chose the regional road traffic network composed of Yatai Street, Minkang Road, Xisandao Street, and Dongsandao Street in Jilin City as the research object (the original data of the traffic network comes from "Research on key nodes and road section identification methods of urban road traffic networks" [19]). Then we ranked the importance of nodes at 10 intersections in the study area and used the traffic data during the morning rush hour $(7: 30-8: 30)$ on December 24, 2013 (Tuesday) as an example to verify the evaluation method effectiveness.

3.1. Regional Traffic Network Construction. The regional traffic network is an area composed of $m, i, h, d, a, b, l$, and $n$. Among them, $m, i$, and $h$ are one-way lines from northeast to southwest; $g$ is one-way lines from southwest to northeast, which is no traffic flow in the opposite direction. The network includes 10 intersections, and the road network structure is shown in Figure 1.

Among them, the inbound traffic flow of each network node is shown in Table 1.

\subsection{Evaluation of Importance of Traffic Nodes Based on Im-} proved Virtual Input-Output Method. Suppose the saturation of a specific lane $j$ at an intersection is $x_{j}$, and $n$ is the number of lanes at the intersection, then the average saturation $x=(1 / n) \sum_{j=1}^{n} x_{j}$ of the entire intersection and the saturation of each intersection are calculated as shown in Table 2.

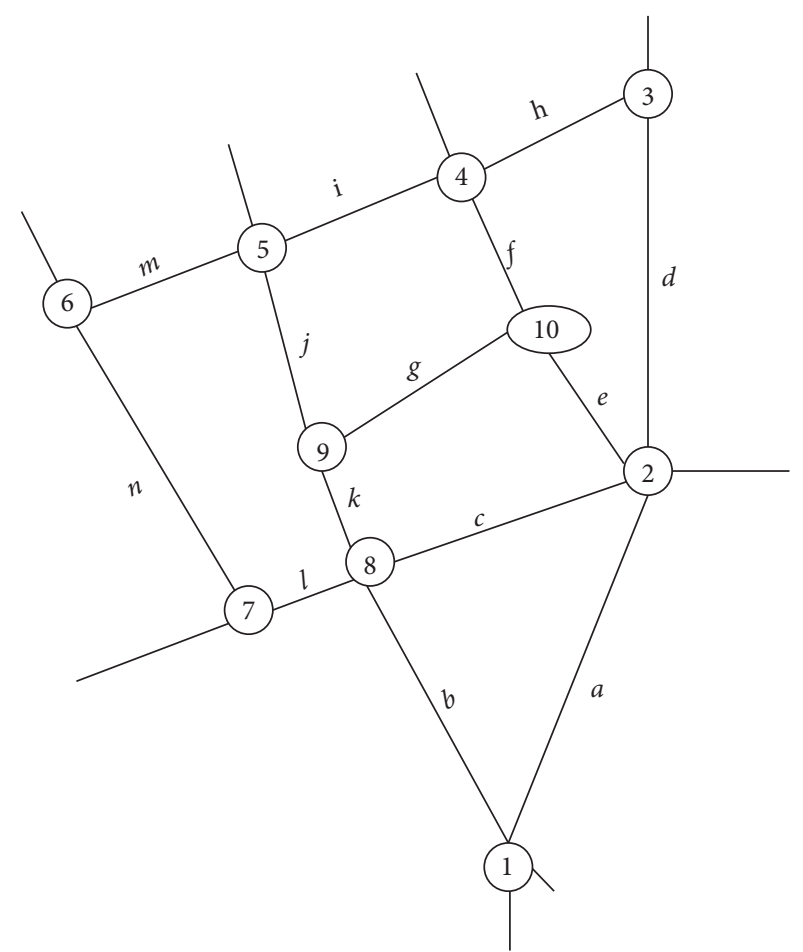

Figure 1: Experimental traffic network structure.

By using the improved virtual input-output method, an urban traffic network system was established. Each node of this network system is represented by each department in the input-output model. The flow of two adjacent nodes has represented the value of the intermediate product provided to the other party by the department in the input-output table. After that, the virtual node $n$ is introduced. Then, by constructing the Leontief inverse matrix, the sensitivity coefficient of the nodes in the urban traffic network can be calculated. Then, the virtual nodes in this experiment were deleted because they only participated in the calculation process in this experiment which had no practical significance. The inductance coefficient after excluding the virtual node in this experiment is shown in Table 3.

It can be known from the definition of the inductance coefficient that the sensitivity of a node represents the importance of this node in this system. The greater the inductance of a node, the more susceptible the node is to the traffic of other nodes in the urban transportation network, and the increase in traffic in the transportation network is more likely to cause pressure on the node. In this experiment, the inductance coefficients of nodes $1,2,7$, and 8 exceed 1 , which is greater than the average inductance coefficient of the whole network. It shows that in this experimental road network, when the traffic volume of the whole network increases, the traffic of these four nodes would be increased significantly. And the importance of these four nodes in the urban transportation network is relatively higher.

In the actual situation, node 2 is the intersection of the three main roads $a, c$, and $d$ in the experimental road network. And field research by data observers found that the 
TABLE 1: Experimental traffic network node import flow.

\begin{tabular}{|c|c|c|c|c|c|c|}
\hline \multirow{2}{*}{ Node } & \multicolumn{6}{|c|}{ Flow (veh) } \\
\hline & South & North & Southeast & Northeast & Southwest & Northwest \\
\hline 1 & 2576 & 1417 & 746 & & & 914 \\
\hline 2 & 1484 & 797 & & 2544 & 1848 & 838 \\
\hline 3 & 885 & 951 & & 598 & & \\
\hline 4 & & & 65 & 1028 & & 1054 \\
\hline 5 & & & 1856 & 952 & & 90 \\
\hline 6 & & & 742 & 523 & & 486 \\
\hline 7 & & & & 2329 & 1583 & 1287 \\
\hline 8 & & & 954 & 2798 & 2593 & 78 \\
\hline 9 & & & 1105 & & 245 & 98 \\
\hline 10 & & & 16 & 156 & 180 & 637 \\
\hline
\end{tabular}

TABLE 2: Experimental traffic network node saturation.

\begin{tabular}{lllllllllll}
\hline Node & 1 & 2 & 3 & 4 & 5 & 6 & 7 & 8 & 9 & 10 \\
\hline Saturation & 0.58 & 0.85 & 0.69 & 0.54 & 0.59 & 0.68 & 0.74 & 0.53 & 34 & 0.46 \\
\hline
\end{tabular}

TABLE 3: Inductance coefficient of nodes in experimental traffic network.

\begin{tabular}{llllllllll}
\hline Node & 1 & 2 & 3 & 4 & 5 & 6 & 7 & 8 & 9 \\
\hline Inductance coefficient & 1.198 & 1.59 & 0.514 & 0.455 & 0.612 & 0.37 & 1.099 & 1.311 & 0.306 \\
Rank & 3 & 1 & 6 & 7 & 5 & 8 & 4 & 2 & 9 \\
\hline
\end{tabular}

morning peak traffic congestion was also the first to occur at node 2 [6]. Also, both nodes 1 and 8, which are quite different from the other two ranking methods, are connected to node 2 with the highest importance. At the same time, the saturation of node 2 is the highest in the entire network, and the increase in the traffic flow of node 2 will inevitably have an overflow effect on neighboring nodes 1 and 8, making it more vulnerable in the road network. It shows that the importance of node 1 and node 8 is reasonable from the perspective of road network dynamics.

\section{Analysis and Verification of Robustness of Traffic Network after Node Failure under Deliberate Attack}

4.1. Network Robustness Analysis. During the operation of the urban transportation network, an emergency occurs on the supply side or the demand side, which means a node of the transportation network within the affected area fails. In this article, these connected edges will be removed; then part of the path will be interrupted. So the traffic network is considered robust when an abnormal situation occurs, and the traffic network can still meet the demands of passenger flow.

Computing network robustness is generally divided into two supply strategies: the deliberate attack and the random attack. This article mainly analyzes deliberate attacks, which means purposeful attacks on nodes or edges in the network. Generally, global efficiency is used as an important evaluation index, in the measurement standard of network robustness when a node fails [20]. The global efficiency $E$ of the network can be expressed as in the following equation:

$$
E=\frac{1}{N(N-1)} \sum_{i \neq j} e_{i j}
$$

where $e_{i j}$ is the efficiency between node pairs. When calculating the robustness of the traffic network under node failure, the passenger flow per unit time between nodes can be used to express the efficiency between node pairs $e_{i j}$, as shown in the following equation:

$$
e_{i j}=\frac{f_{i j}}{t_{i j}},
$$

where $f_{i j}$ is the product of the number of cars between nodes $i$ and $j$ and the average passenger capacity, and $t_{i j}$ is 3600 seconds. In this case, the connectivity of the network would decrease, and the global efficiency would also decrease when a node in the network receives an attack. The evaluation index of robustness is the ratio of the global efficiency of the network after the attack to the initial global efficiency of the network, as shown in the following formula:

$$
R_{E}=\frac{E^{\prime}}{E_{0}} .
$$

4.2. Experimental Traffic Network Robustness after Node Failure under Deliberate Attack. In order to verify the effectiveness of the key node identification of the transportation network based on the virtual input-output method in this study, each node is selected one node at a time according to the order of node importance, according to the inductance coefficient (importance) of the nodes in the experimental transportation network in Table 3. After 
TABLE 4: The robustness analysis results of experimental traffic network under deliberate attack.

\begin{tabular}{lcccc}
\hline Node & $E_{0}$ & $E^{\prime}$ & $R_{E}$ & Rank \\
\hline 2 & 0.1962 & 0.0988 & 0.5036 & 0.6033 \\
8 & 0.1962 & 0.1184 & 0.8123 & 2 \\
1 & 0.1962 & 0.1594 & 0.7265 & 4 \\
7 & 0.1962 & 0.1425 & 0.8650 & 3 \\
5 & 0.1962 & 0.1697 & 0.8933 & 7 \\
3 & 0.1962 & 0.1753 & 0.8944 & 8 \\
4 & 0.1962 & 0.1755 & 0.8995 & 9 \\
6 & 0.1962 & 0.1765 & 0.8694 & 6 \\
9 & 0.1962 & 0.1706 & 0.9316 & 10 \\
\hline
\end{tabular}

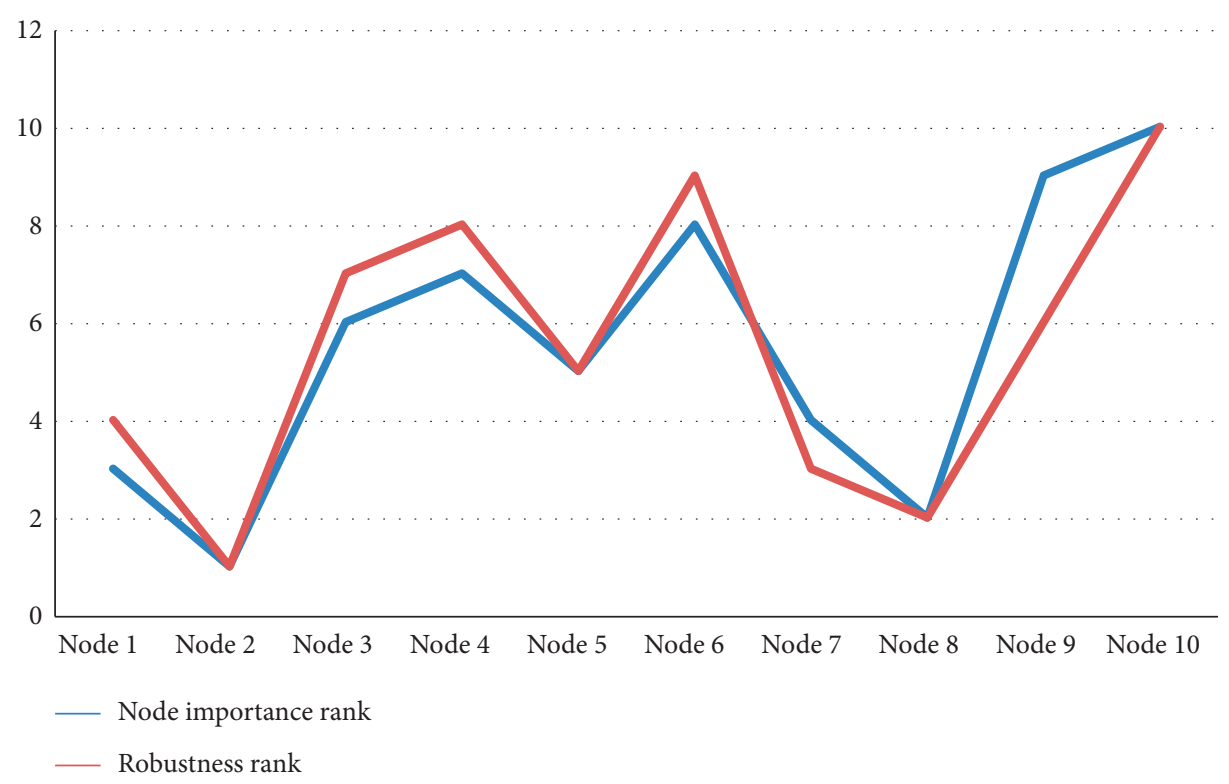

FIGURE 2: Comparison of node importance ranking and robust ranking.

deliberately attacked, the experimental traffic network robustness results after the attack are shown in Table 4:

According to the results of robustness analysis, it is basically consistent that the order of node importance with the order of global efficiency change of the experimental traffic network after a deliberate attack. Among them, the most important node 2 decreases the global effectiveness the most after being attacked, and the second most important node 1 decreases the global effectiveness after being attacked. The rest are basically the same (Figure 2), except that the importance of nodes 7, 5, and 9 is different from the global effectiveness reduction ranking after being attacked (the difference is small).

\section{Conclusion}

This paper studies a new method to identify key nodes in the transportation network based on the improved virtual inputoutput method. Firstly, each node in the transportation network is regarded as each department in the input-output model, and virtual nodes are introduced to establish an urban transportation network structure with traffic. Then the Leontief inverse matrix is constructed to calculate the inductance coefficient of the traffic network node, which can evaluate the importance of the node. After that, according to the evaluated node importance ranking and the measurement index of the global efficiency changing, the different nodes of importance are judged in turn on the robustness of the traffic network after failure. For this new method, we carry out empirical research based on the traffic volume data of an experimental road network during peak hours. Then, results show that the improved virtual input-output method applied to the importance of urban traffic road network node recognition has high accuracy and has the advantages of fast calculation and simple process. This research provides a new solution for the rational allocation of urban traffic management resources and ensuring overall smooth traffic.

The method in this study has certain theoretical significance and engineering practical value. However, the data adopted in this paper are collected by related information in Jilin. Therefore, it is necessary to collect more data in other provinces to verify this method. 


\section{Data Availability}

All data generated or used during the study appear in the submitted article.

\section{Conflicts of Interest}

The authors declare that they have no conflicts of interest.

\section{Acknowledgments}

The authors acknowledge financial support from Soft Science Research Program of Shaanxi Province (2019KRM101), Scientific Research Program Funded by Shaanxi Province Education Department (17 JK0306), Scientific Research Foundation for Doctors of XPU (3100401016), and Nature Science Fund of Qinghai Province (No. 2020-ZJ-736).

\section{References}

[1] S. White and P. Smyth, "Algorithms for estimating relative importance in networks," in Proceedings of the Ninth ACM SIGKDD International Conference on Knowledge Discoveryand Data Mining, pp. 268-272, New York, NY, USA, August 2003.

[2] D. B. Chen, L. Y. Lu, and M. S. Shang, "Identifying influential nodes in complex net-works," Physical A Statistical Mechanics \& Its Applications, vol. 391, no. 4, pp. 177-178, 2012.

[3] M. Kitsak, L. K. Gallos, S. Havlin et al., "Identification of influential spreads in complex networks," Nature Physics, vol. 6, pp. 888-893, 2010.

[4] J. Chen and L. F. Sun, "Evaluation of node importance in complex networks," Journal of Southwest Jiaotong University, vol. 452, pp. 26-29, 2009.

[5] H. Yu, Z. Liu, and Y. J. Li, "Comprehensive evaluation method of node importance in complex networks based on multiattribute decision making," Journal of Physics, vol. 2, no. 62, 2013.

[6] M. Coscia, A. G. Lievano, J. Mcnerney, and F. Neffke, "The node vector distance problem in complex networks," ACM Computing Surveys (CSUR), vol. 53, no. 6, 2020.

[7] H. Q. Song, J. Guo, and G. Liu, "Evaluation of urban road importance and automatic road network synthesis method based on complex network," Surveying and Mapping Engineering, vol. 26, no. 1, pp. 8-12, 2017.

[8] H. Zhang and Y. G. Yao, "An integrative vulnerability evaluation model to urban road complex network," Wireless Personal Communications, vol. 107, pp. 193-204, 2019.

[9] C. B. Li, L. Wei, and F. X. Li, "Study on vulnerability of complex transportation network of Urban Agglomeration Based on attack strategy," Journal of Highway and Transportation Research and Development, vol. 34, no. 3, pp. 101$108,2017$.

[10] B. Q. Cheng, Y. H. Wei, W. Zhang et al., "Evolutionary game simulation on government incentive strategies of prefabricated construction: a system dynamics approach," Complexity, vol. 2020, no. 11, Article ID 8861146, 2020.

[11] W. Leontief, Input-Output Economics, pp. 19-40, Oxford University Press, Oxford, UK, 1986.

[12] G. P. Yang, Modern Industrial Economics, Fudan University Press, ShangHai, China, 2005.

[13] W. H. Zhu, K. Liu, and X. Y. Yan, "Virtual external input and output analysis method of identifying key nodes of flow network," Journal of The University of Electronic Science and Technology, vol. 47, no. 2, pp. 292-297, 2018.

[14] Z. Shen, L. Yang, J. Pei et al., "Interrelations among scientific fields and their relative influences revealed by an input-output analysis," Journal of Informetrics, vol. 10, no. 1, pp. 82-97, 2016.

[15] B. Q. Cheng, J. W. Li, V. W. Y. Tam, M. Yang, and D. Chen, "A BIM-LCA approach for estimating the greenhouse gas emissions of large-scale public buildings: a case study," Sustainability, vol. 12, no. 2, 2020.

[16] D. H. Tang, Study on Complex Input and Output Flow Network, China University of Science and Technology, Taipei, Taiwan, 2014.

[17] H. H. Chen, H. J. Li, Y. G. Wang, and B. Q. Cheng, "A Comprehensive assessment approach for water-soil environmental risk during railway construction in ecological fragile region based on AHP and MEA," Sustainability, vol. 12, no. 19, 2020.

[18] L. Y. Lu, Y. C. Zhang, and C. H. Yeung, "Leaders in social networks, the delicious case," Plos One, vol. 6, no. 6, 2011.

[19] W. Wang, Study on Key Nodes and Road Segment Identification Methods of Urban Road Traffic Network, Jilin University, Changchun, China, 2015.

[20] V. Latora and M. Marchiorim, "Efficient behavior of smallworld networks," Physical Review Letters, vol. 87, no. 19, 2001. 